\title{
La pharmacopée ultramarine enfin réhabilitée : entre histoire et modernité
}

L'histoire commence durant la période coloniale; les nègres libres ou esclaves avaient l'interdiction d'exercer la médecine et la chirurgie, mais aussi de dispenser les plantes sous quelque forme que ce soit sous peine de châtiment corporel pour les esclaves et de déchéance de leurs droits sur leurs esclaves pour les maîtres (arrêt du Conseil souverain du 8 mars 1799), les colons craignant d'être empoisonnés.

Telles sont les informations historiques que le Dr Henry Joseph et moi-même avions découvertes lors de nos recherches.

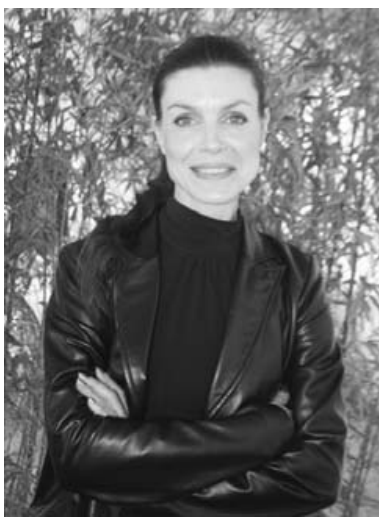

Or, l'histoire du droit pharmaceutique nous enseigne que c'est par la loi du 11 avril 1803 qu'est prévue la mise en place du premier codex.

Ce premier codex paraitra en 1818 , longtemps avant l'abolition définitive de l'esclavage en 1848, expliquant que les plantes françaises d'Outre-mer en furent totalement exclues.

À ce jour, sur plus de 400 plantes figurant à la pharmacopée française, seules 29 plantes domiennes y figurent, plantes qui s'apparentent davantage à des épices et aromates qu'à des plantes médicinales. Et encore, nous compterons dans cette - liste Senna alata et Lippia alba qu'avec le Pr Bourgeois, le Pr Portecop et le Dr Joseph, nous réussîmes à faire entrer après quatre ans de démarches auprès de l'Afssaps.

Or, un pharmacien d'officine, pour délivrer des plantes ou préparations magistrales à base de plantes, doit se référer à la pharmacopée française et/ou européenne. Seules les plantes inscrites à la pharmacopée française peuvent être yendues légalement et déontologiquement, avec toutes les conséquences que cela peut entraîner dans la chaîne de soins.

En conséquence, un pharmacien d'officine d'Outre-mer, en raison du défaut d'inscription des plantes domiennes à la pharmacopée française, ne peut proposer à la vente les plantes de sa propre région. C'est pourquoi, avec 
l'Aplamedarom (Association pour la promotion des plantes médicinales et aromatiques de Guadeloupe), le Dr Joseph, les Prs Bourgeois et Portecop, nous avons décidé, en 2001, d'entamer tout un travail juridicoscientifique afin de faire modifier le code de la santé publique.

Rarement une proposition de réforme aura été votée autant de fois : le Conseil des ministres vote le 7 mars 2003 la réforme proposée, le Sénat et l'Assemblée nationale entérinent également le projet de modification du code de la santé publique dans le cadre de la loi de programme en juillet 2003, mais son exécution n'est pas prise dans les 18 mois, nouvelle adoption du principe de réforme suite au Grenelle de l'environnement en juin 2008, vote du principe de la réforme dans la loi Grenelle I en octobre 2008, mais retrait de la réforme dans la loi Grenelle II.

Décidant d'alerter les parlementaires antillais à qui nous rendons hommage (notamment Mme Lucette Michaux-Chevry, MM. Victorin Lurel et Serge Letchimy) pour leur opiniâtreté lors des débats à l'Assemblée nationale, après huit années de combat acharné, c'est finalement le 8 avril 2009 que cette réforme du code de la santé publique est adoptée mettant un point final historique à cette discrimination.

Il reste maintenant à procéder à l'application de cette réforme par la mise en place des décrets d'application en vue de permettre notamment une meilleure représentativité, dans les instances de l'Afssaps, des scientifiques compétents en matière de pharmacopée ultramarine, mais également de permettre une prise en compte des travaux menés par l'équipe Tramil, composée de 200 chercheurs qui, sans relâche, depuis 25 ans, produit des travaux de référence dans le domaine de la pharmacopée caribéenne.

Nul doute que nous serons vigilants...

I. Robard

Docteur en droit, chargée d'enseignement en faculté de droit. 